\title{
Forceless Sadowsky strips are spherical
}

\author{
E. L. Starostin* and G. H. M. van der Heijden ${ }^{\dagger}$ \\ Department of Civil, Environmental \& Geomatic Engineering, University College London, \\ Gower Street, London WC1E 6BT, United Kingdom
}

(Received 2 May 2017; published 8 February 2018)

\begin{abstract}
We show that thin rectangular ribbons, defined as energy-minimizing configurations of the Sadowsky functional for narrow developable elastic strips, have a propensity to form spherical shapes in the sense that forceless solutions lie on a sphere. This has implications for ribbonlike objects in (bio)polymer physics and nanoscience that cannot be described by the classical wormlike chain model. A wider class of functionals with this property is identified.
\end{abstract}

DOI: 10.1103/PhysRevE.97.023001

\section{INTRODUCTION}

Understanding the configurations and stresses of biopolymers lying on a surface is important in a number of biomolecular processes, including the packing of DNA inside viral capsids [1], cytokinesis in animal and yeast cells during which mainly membrane-bound actin filaments provide the forces necessary for cell division [2], and cell wall synthesis in bacteria $[3,4]$. Graphene nanoribbons have also been studied on surfaces [5] with a view to assembling ribbonlike nanomaterials with desirable properties.

A classical theoretical approach to the study of such filamentous objects is to use the wormlike chain (WLC) model [6] in which the polymer is assumed to have only entropic bending elasticity (characterising the persistence length). For biopolymers, like DNA, that also have torsional elasticity, the torsional directed walk or rodlike chain (RLC) is a more appropriate model $[7,8]$.

If the biopolymer is ribbonlike, i.e., much thinner than it is wide, then the polymer essentially behaves as a thin sheet. Such sheets (e.g., paper) tend to deform isometrically, i.e., without stretching. The deformed shape of an intrinsically flat ribbon is therefore part of a developable surface. Accordingly, an elastic developable strip model has been proposed for ribbonlike filaments $[9,10]$. Since developable surfaces can be completely reconstructed from the strip's deformed centerline, the problem of finding equilibrium solutions for such strips can be formulated as a variational problem on a space curve for an energy functional in which the width $2 w$ appears merely as a parameter [11,12]. In the limit of a narrow strip, $w \rightarrow 0$, this functional reduces to the Sadowsky functional $[13,14]$,

$$
\int \kappa^{2}\left(1+\eta^{2}\right)^{2} d s
$$

where $s$ is arc length, $\kappa$ is the curvature, $\eta=\tau / \kappa$, and $\tau$ is the torsion of the curve. The straight generators of the surface make an angle $\beta=\arctan (1 / \eta)$ with the tangent to the centerline (see Fig. 1). More precisely, the Sadowsky functional (1) is valid

\footnotetext{
*e.starostin@ucl.ac.uk

${ }^{\dagger}$ g.heijden@ucl.ac.uk
}

in the limit $\left|w \eta^{\prime}\right| \ll 1$, which means that $w$ does not have to be small if the angle the generator makes with the centerline varies only very gradually with arc length $s$. A strip deformed in the shape of a cylinder, for example, which has $\eta^{\prime}=0$, is described by Eq. (1) (for arbitrary $w$ ). An asymptotic analysis of the validity of functional (1), in terms of geometrical and load parameters, is given in [15]. The Sadowsky functional originated in mechanical studies of Möbius strips [13,14]. The functional is a singular limit of the finite-width functional near inflection points of the centerline [11,16,17].

There is a long line of research, stretching back to Manning's work [18], on equilibrium paths of elastic lines on curved surfaces. Generally, a filament lying on a physical surface requires a distributed reaction force from the surface onto the (intrinsically straight) filament. The surface has to be stiff enough to provide the required force, which will increase with the curvature of the surface. These external forces acting on the filament induce internal forces and hence stresses in the material. For the important ideal model problem of a spherical surface, for instance, both the WLC and RLC model require a reaction force [18-21]. Here we show that, remarkably, Sadowsky strips are spherical if forceless, meaning that no distributed force is required to constrain them to a spherical surface. So no tensile or compressive stresses need to be sustained by the material. We like to speculate that nature may have found ways to exploit this fact in the interaction between biofilaments and surfaces or vesicles. By contrast, we mention the well-known fact that forceless solutions of the Kirchhoff rod (RLC) are helices (with the straight rod and the ring as degenerate states), while for the special case of the Euler elastica (WLC) they are rings (or straight rods).

In fact, the Sadowsky functional is just the simplest functional of a family of functionals whose equilibrium curves are spherical. Therefore, in the next section we start with the more general formulation of a geometric variational problem on a space curve.

\section{GEOMETRIC VARIATIONAL PROBLEMS ON SPACE CURVES}

A space curve $\gamma:[0, L] \rightarrow \mathbb{R}^{3}$ without inflection points is completely characterized (up to Euclidean motions) by its 


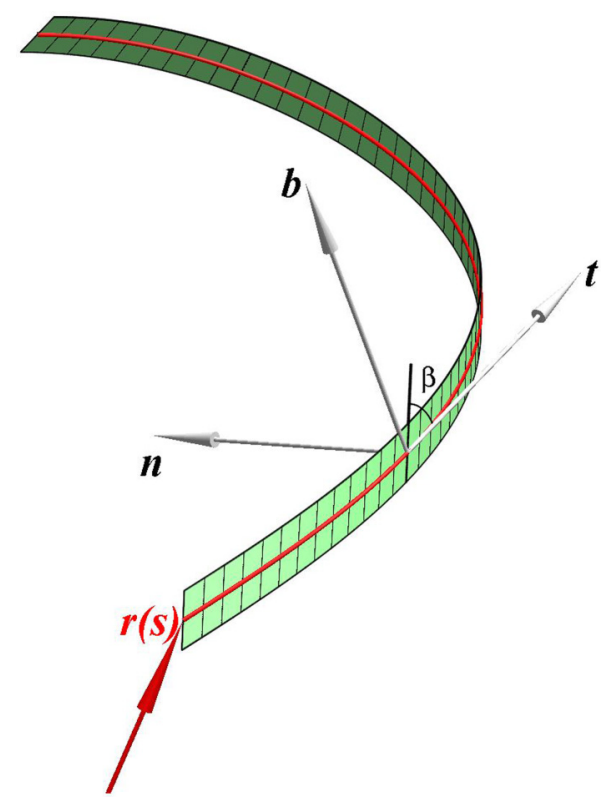

FIG. 1. A developable strip is made up of straight generators in the rectifying plane of tangent $\boldsymbol{t}$ and binormal $\boldsymbol{b}$ to the centerline $\boldsymbol{r}$. The generators make an angle $\beta$ with the tangent. $\boldsymbol{n}$ is the principal normal.

curvature $\kappa(s)(>0)$ and the ratio $\eta(s)=\tau(s) / \kappa(s)$, where $\tau(s)$ is the torsion. We consider functionals on such curves of the form

$$
U(\gamma)=\int_{0}^{L} l(\kappa, \eta) d s
$$

Functionals of this type appear in a range of applications. For instance, the classical case $l=\kappa^{2}$ gives the Euler elastica used as a model for the bending of elastic rods or polymers. The case $l=(A \kappa+B \eta) \kappa$ gives the isotropic Kirchhoff rod having both bending and torsional stiffness [22], while $l=\left(A+B \eta^{2}\right) \kappa^{2}$ describes a thin strip whose material frame is locked to the Frenet frame and which therefore bends only about a single principal axis [23]. The linear function $l=A+B \kappa+C \tau$, meanwhile, which gives rise to generalized (Lancret) helices (having constant $\eta$ ), has been proposed for protein chains [24]. Functionals $U$ as in Eq. (2) also appear in the localized induction hierarchy, an idealized model of the evolution of vortex filaments in three-dimensional inviscid incompressible fluids [22], and its generalizations [25]. The kinematics of space curves is furthermore related to integrable systems such as the nonlinear Schrödinger equation and the modified Korteweg-de Vries equation [26,27].

Critical points of $U$ satisfy the following equilibrium conditions [28]: (a) balance equations for the components of the internal force $\mathrm{F}=\left(F_{t}, F_{n}, F_{b}\right)^{\top}$ and moment $\mathrm{M}=$ $\left(M_{t}, M_{n}, M_{b}\right)^{\top}$ expressed in the Frenet frame $\{\boldsymbol{t}, \boldsymbol{n}, \boldsymbol{b}\}$ (tangent, principal normal, and binormal):

$$
\begin{gathered}
\mathrm{F}^{\prime}+\omega \times \mathrm{F}=\mathbf{0}, \\
\mathrm{M}^{\prime}+\omega \times \mathrm{M}+\mathrm{t} \times \mathrm{F}=\mathbf{0},
\end{gathered}
$$

where $\boldsymbol{\omega}=(\kappa \eta, 0, \kappa)^{\top}$ is the curvature (Darboux) vector in the Frenet frame and $t=(1,0,0)^{\top}$, and (b) the "constitutive" relations

$$
M_{t}=\frac{1}{\kappa} \frac{\partial l}{\partial \eta}, \quad M_{b}=\frac{\partial l}{\partial \kappa}-\frac{\eta}{\kappa} \frac{\partial l}{\partial \eta} .
$$

The force vector is a constant vector in space, and $F^{2}$ and F . M are first integrals of Eqs. (3) and (4). A further conserved quantity is the Hamiltonian given by

$$
H=\kappa \frac{\partial l}{\partial \kappa}-l+F_{t} .
$$

The equations can alternatively be derived through EulerPoincaré reduction [11] or by direct variation [29-31].

\section{FORCELESS SPACE CURVES}

We now consider the special case of forceless solutions, $\mathbf{F}=\mathbf{O}$. For such solutions the moment vector is conserved and the Hamiltonian becomes $H=\kappa l_{\kappa}-l$. By generalizing from some of the integrands $l$ in Eq. (2) reviewed above, we let $l$ be the product of two factors:

$$
l(\kappa, \eta)=\kappa^{n} p(\eta)
$$

where $n$ is an arbitrary number (not necessarily an integer) and $p(\eta) \in C^{3}$ is an arbitrary positive function of its single argument $\eta$. The corresponding Hamiltonian is $H=(n-$ 1) $\kappa^{n} p(\eta)=h=$ const. For $n \neq 0,1$, we have

$$
\kappa=\left(\frac{h}{(n-1) p(\eta)}\right)^{1 / n}>0 .
$$

The constitutive equations (5) allow us to solve for two components of the moment vector,

$$
\begin{aligned}
M_{t} & =\left[\frac{h}{(n-1) p}\right]^{1-1 / n} p_{\eta}, \\
M_{b} & =\left[\frac{h}{(n-1) p}\right]^{1-1 / n}\left(n p-\eta p_{\eta}\right) .
\end{aligned}
$$

The remaining component is found by differentiating $M_{t}$ and using the first component of Eq. (4):

$$
M_{n}=\left(\frac{h}{n-1}\right)^{1-2 / n} p^{2 / n-2}\left[p p_{\eta \eta}+\left(\frac{1}{n}-1\right) p_{\eta}^{2}\right] \eta^{\prime} .
$$

It is easy to check that the above expressions satisfy the third component of Eq. (4) identically and that the second component can be written as

$$
\begin{gathered}
A_{2} \eta^{\prime \prime}+A_{1} \eta^{2}+\left(\frac{h}{n-1}\right)^{2 / n} A_{0}=0 \\
A_{2}=p^{2 / n}\left[p p_{\eta \eta}+\left(\frac{1}{n}-1\right) p_{\eta}^{2}\right], \\
A_{1}=p^{2 / n-1}\left[p^{2} p_{\eta \eta \eta}+\left(\frac{4}{n}-3\right) p p_{\eta} p_{\eta \eta}+2\left(\frac{1}{n}-1\right)^{2} p_{\eta}^{3}\right],
\end{gathered}
$$

$$
A_{0}=p\left[\left(1+\eta^{2}\right) p_{\eta}-n \eta p\right]
$$


We now recall the criterion for a curve to be spherical.

Theorem [32]. The necessary and sufficient conditions for a $C^{4}$ regular curve $\boldsymbol{r}(s)$ to lie on a sphere are

(i) the curvature $\kappa$ does not vanish (hence the torsion $\tau$ is defined),

(ii) there exists a $C^{1}$ function $f(s)$ such that

$$
f \tau=\left(\frac{1}{\kappa}\right)^{\prime}, \quad f^{\prime}+\frac{\tau}{\kappa}=0 .
$$

The curve satisfying this criterion lies on a sphere of radius $R=\sqrt{\kappa^{-2}+f^{2}}$. Note that the above theorem does not require nonvanishing torsion of the curve.

Differentiating the expression for the curvature Eq. (7) we obtain

$$
\left(\frac{1}{\kappa}\right)^{\prime}=\frac{1}{n}\left(\frac{n-1}{h}\right)^{1 / n} p^{1 / n-1} p_{\eta} \eta^{\prime} .
$$

We define $f=\frac{1}{n \kappa}\left(\frac{n-1}{h}\right)^{1 / n} p^{1 / n-1} \frac{p_{\eta}}{\eta} \eta^{\prime}$, assuming that $\lim _{\eta \rightarrow 0} \frac{p_{\eta}(\eta)}{\eta}$ exists and is finite. After substitution of $\kappa$ this becomes $f=\frac{1}{n}\left(\frac{n-1}{h}\right)^{2 / n} p^{2 / n-1} \frac{p_{\eta}}{\eta} \eta^{\prime}$. Differentiating $f$ with respect to $s$ and inserting the result into the equation $f^{\prime}+\eta=$ 0 , we arrive, after simplification, at a second-order equation for $\eta$ :

$$
\begin{gathered}
B_{2} \eta^{\prime \prime}+B_{1} \eta^{2}+\left(\frac{h}{n-1}\right)^{2 / n} B_{0}=0, \\
B_{2}=p^{2 / n-1} p_{\eta} \eta, \\
B_{1}=p^{2 / n-2}\left[p\left(p_{\eta \eta} \eta-p_{\eta}\right)+\left(\frac{2}{n}-1\right) p_{\eta}^{2} \eta\right], \\
B_{0}=n \eta^{3} .
\end{gathered}
$$

We can now ask the question, for what $p(\eta)$ does Eq. (12) coincide with Eq. (8)? If it does, then solutions of Eq. (8) are spherical. To answer the question, we match the coefficients of our two equations, which gives two new equations:

$$
\begin{aligned}
& A_{2} B_{0}=A_{0} B_{2}, \\
& A_{1} B_{0}=A_{0} B_{1} .
\end{aligned}
$$

These are two nonautonomous ordinary differential equations for $p(\eta)$.

Equation (16) simplifies to

$$
\eta^{2} p p_{\eta \eta}-\left(\eta^{2}+\frac{1}{n}\right) p_{\eta}^{2}+\eta p p_{\eta}=0 .
$$

Its general solution is

$$
p(\eta)=C\left(\eta^{2}+\frac{N}{n}\right)^{N}
$$

where $C$ and $N$ are integration constants. Note that the ratio $\frac{p_{\eta}(\eta)}{\eta}$ is well defined for $\eta=0$. Direct substitution of the above $p(\eta)$ into the second condition Eq. (17) reveals that it is satisfied only for $N=n$ (the arbitrary prefactor constant $C$ is clearly of no importance). Thus, we conclude that all forceless inflectionfree minimizers of the functional $l(\kappa, \eta)=\kappa^{n} p(\eta), n \neq 0,1$, are spherical only for

$$
l(\kappa, \eta)=C \kappa^{n}\left(1+\eta^{2}\right)^{n}, \quad C=\text { const . }
$$

The radius of the sphere is $R=\left|\frac{n-1}{n} \frac{M}{h}\right|$, where $M^{2}=M_{t}^{2}+$ $M_{n}^{2}+M_{b}^{2}>0$. A special analysis reveals that for $n=1$, Eq. (18) gives, among other solutions, arbitrary planar curves ( $\eta=0$ ). For $n=0$, Eq. (18) is trivial, but Eq. (6) gives Lancret helices, for arbitrary nonconstant $p$.

\section{THE SADOWSKY FUNCTIONAL - FORCELESS STRIP SOLUTIONS}

For $n=2$ in Eq. (18) we obtain the Sadowsky functional Eq. (1):

$$
U_{S}(\gamma)=\int_{0}^{L} \kappa^{2}\left(1+\eta^{2}\right)^{2} d s .
$$

For forceless strips Eqs. (3), (4), and (5) reduce to

$$
\begin{gathered}
M_{t}^{\prime}=\kappa M_{n}, \quad M_{n}^{\prime}=\kappa \eta M_{b}-\kappa M_{t}, \quad M_{b}^{\prime}=-\kappa \eta M_{n}, \\
M_{t}=4 \kappa \eta\left(1+\eta^{2}\right), \quad M_{b}=2 \kappa\left(1-\eta^{4}\right),
\end{gathered}
$$

while the Hamiltonian is

$$
H=\kappa^{2}\left(1+\eta^{2}\right)^{2} .
$$

The remaining normal component of the moment may be found from the first (or third) equation in (20) and (22):

$$
M_{n}=4\left(1+\eta^{2}\right) \eta^{\prime}
$$

Combination with the second equation in (20) and again (22) then gives

$$
2\left(1+\eta^{2}\right) \eta^{\prime \prime}+4 \eta \eta^{2}+h \eta=0,
$$

where $h$ is the value of the Hamiltonian. The theorem above tells us that solutions of this equation represent spherical curves, i.e., centerlines of narrow forceless rectangular strips are spherical curves. The radius of the sphere equals $R=\frac{M}{2 h}$.

Integrating Eq. (24) once gives the moment first integral

$$
G\left(\eta, \eta^{\prime}\right):=4\left(1+\eta^{2}\right)^{2}\left(4 \eta^{\prime 2}+h\right)=M^{2} .
$$

Analysis of the derivatives of $G\left(\eta, \eta^{\prime}\right)$ reveals that there always exists only one critical point at the origin and that it is always a center point. Therefore, all the orbits in the phase plane are closed (see Fig. 2).

Further integration of Eq. (25) yields

$$
\pm \frac{2}{\sqrt{h}} \int_{0}^{\eta} \frac{1+\eta^{2}}{\sqrt{A^{2}-\left(1+\eta^{2}\right)^{2}}} d \eta=s-s_{0},
$$

where $A^{2}=\frac{M^{2}}{4 h}=h R^{2} \geqslant 1$, the inequality following from Eq. (25). Evaluation of the integral delivers the final equation

$$
\begin{aligned}
& \sqrt{2 A}\left[2 \mathrm{E}\left(\eta \sqrt{\frac{2 A}{(A-1)\left(A+1+\eta^{2}\right)}}, \sqrt{\frac{A-1}{2 A}}\right)\right. \\
& \left.-\mathrm{F}\left(\eta \sqrt{\frac{2 A}{(A-1)\left(A+1+\eta^{2}\right)}}, \sqrt{\frac{A-1}{2 A}}\right)\right]- \\
& -2 \eta \sqrt{\frac{A-1-\eta^{2}}{A+1+\eta^{2}}}= \pm \sqrt{h}\left(s-s_{0}\right),
\end{aligned}
$$




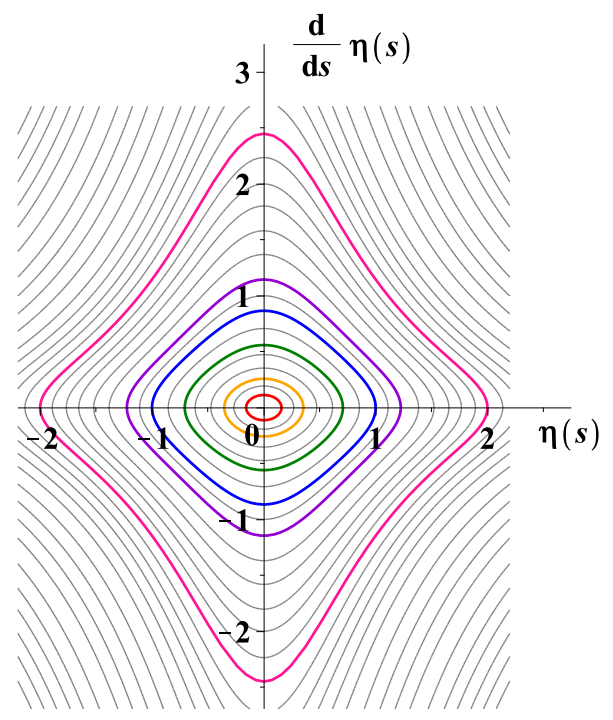

FIG. 2. Phase portrait for Eq. (25) with orbits for $M=2.05$, $2.25,3,4,5$, and 10 (inner to outer) highlighted $(h=1)$.

where $\mathrm{F}(z, k)=\int_{0}^{z}\left(1-k^{2} \sin ^{2} u\right)^{-\frac{1}{2}} d u$ and $\mathrm{E}(z, k)=\int_{0}^{z}(1-$ $\left.k^{2} \sin ^{2} u\right)^{\frac{1}{2}} d u$ are the incomplete elliptic integrals of the first and second kind, respectively (with $k$ the elliptic modulus), and $s_{0}$ is an integration constant. Once this equation is solved for $\eta$, the curvature can be computed as

$$
\kappa=\frac{\sqrt{h}}{1+\eta^{2}} .
$$

(a)
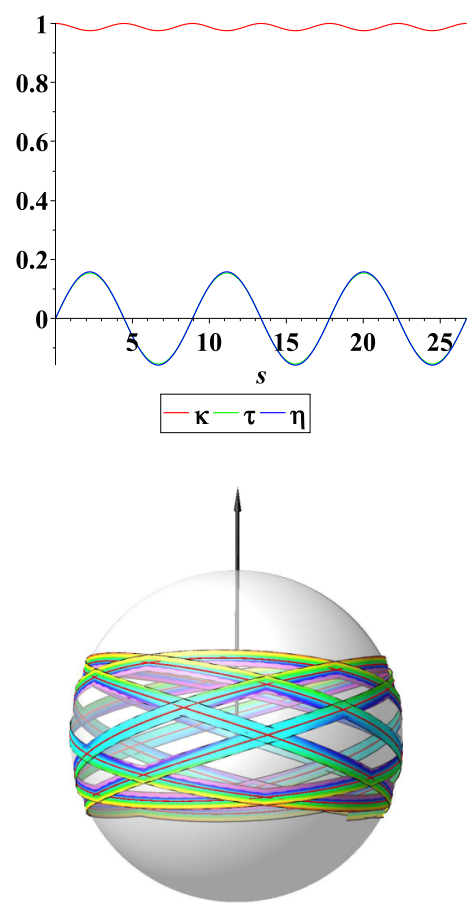

(b)
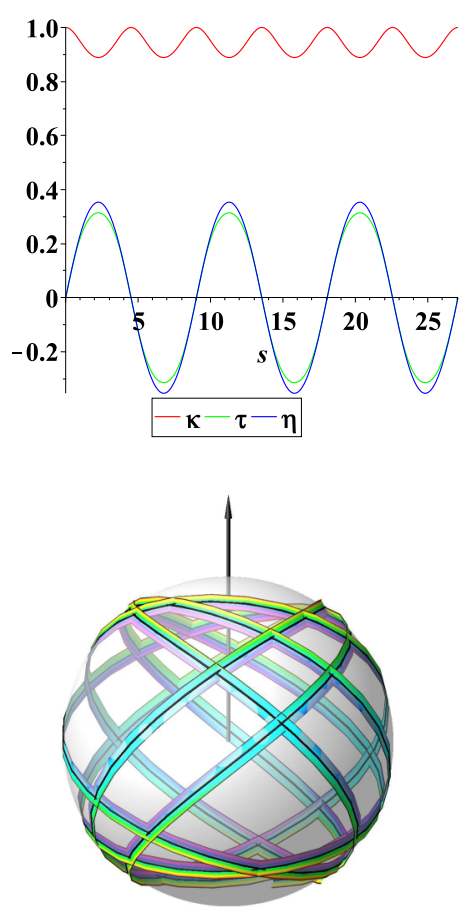

As follows from Eq. (25), $\eta^{\prime}$ goes through a maximum or minimum when $\eta=0$, while $\eta$ goes through a maximum or minimum, $\eta= \pm \sqrt{A-1}$, when $\eta^{\prime}=0$. Using this, the period can be computed from Eq. (26) as

$$
T=4 \sqrt{\frac{2 A}{h}}\left[2 \mathrm{E}\left(\sqrt{\frac{A-1}{2 A}}\right)-\mathrm{K}\left(\sqrt{\frac{A-1}{2 A}}\right)\right],
$$

where $\mathrm{K}(k)$ and $\mathrm{E}(k)$ are the complete elliptic integrals of the first and second kind, respectively. The curvature is then periodic with period $T / 2$. The expression for the Hamiltonian implies that zeros of $\eta$ correspond to maxima of the curvature, $\kappa_{\max }=\sqrt{h}$, while $\eta$ has extrema at points where $\kappa$ has a minimum, $\kappa_{\min }=\frac{\sqrt{h}}{A}=\frac{1}{R}$ (see Figs. 3 and 4). Note that the torsion $\tau$ averaged over a period $T$ is zero. Solutions are therefore achiral.

We also note that the tangential component of the moment is proportional to $\eta: M_{t}=4 \sqrt{h} \eta$. Thus the tangent to the centerline makes an angle with the moment vector with cosine equal to $M_{t} / M=2 \eta / A$. This implies that the tangent to the centerline is oriented orthogonally to the fixed axis of the moment vector at points where $\eta=0$, while the tangent to the centerline is aligned with the moment vector at points where $\eta= \pm A / 2$. Since $-\sqrt{A-1} \leqslant \eta \leqslant \sqrt{A-1}$, the latter occurs at maximum $|\eta|$ if $A=2$, i.e., $\eta= \pm 1$ [Fig. 4(a) gives an example for $h=1, M=4]$.

Shapes of strips on the sphere are shown in Figs. 3 and 4. Here the strips are drawn with a small width to illustrate that they rotate relative to the (imaginary) spherical surface. The angle $\chi$ between the normal to the developable surface of the ribbon at its centerline and the normal to the sphere 
(a)
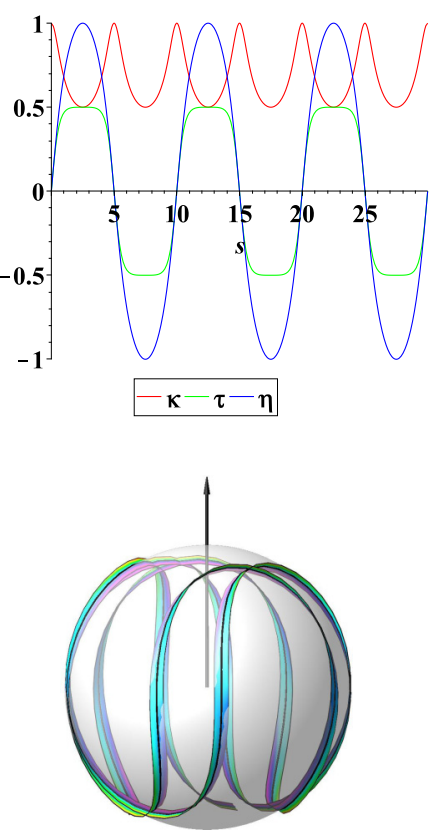

(b)
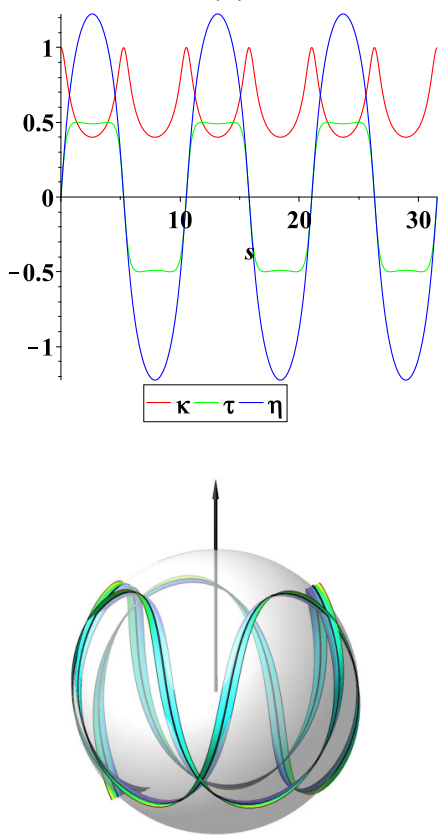

(c)
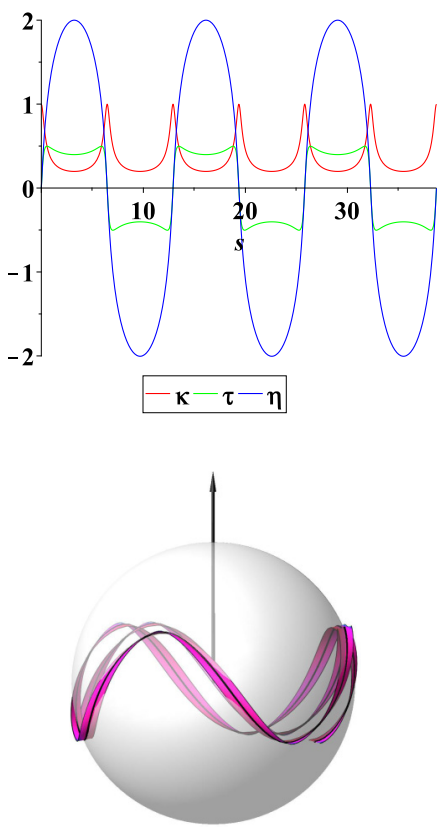

FIG. 4. Continued from Fig. 3 for (a) $M=4(T=9.99339)$, (b) $M=5$ ( $T=10.52595)$, (c) $M=10(T=12.91809)$.

can be found from the equation $\kappa \cos \chi=\frac{1}{R}$. We see that at points of vanishing $\eta$, where the generator is orthogonal to the centerline, this angle reaches its maximum value, while it vanishes at points of maximum $|\eta|$. In the latter case the tangent plane to the ribbon's surface is also tangent to the sphere.

Strips are generally not closed on the sphere, but periodic bounadry conditions (in both space and curvature) could be imposed, which would fix one of the two free parameters $(M, h)$, leaving a one-parameter family of closed solutions. Note that these structures would be closed as a strip, since periodicity of curvature and torsion enforces periodicity of the Frenet frame and alignment of the end generators. They would have high-order spatial symmetry, namely, $D_{n d}$ symmetry ( $n$ being a mode number), with planes of reflection symmetry through the moment vector alternating with axes of $\pi$-rotation symmetry perpendicularly intersecting the central moment axis and transversely intersecting the symmetry planes. Nonclosed (quasiperiodic) strip solutions, meanwhile, have $D_{\infty h}$ symmetry. Structures with either of these symmetry groups must indeed have zero force, as there can neither be a force component in a plane of reflection symmetry nor along an axis of rotation symmetry.

\section{DISCUSSION}

We have shown that a class of energy functionals for elastic filaments, which includes the Sadowsky energy for a narrow strip, has spherical forceless extremals. For the Sadowsky case solutions depend on two parameters: the values of the two first integrals, i.e., the magnitude of the moment $(M)$ and the Hamiltonian $(h)$, which is also the (normalized) bending energy density. The radius of the sphere is $\frac{M}{2 h}$.

The class of functionals with this property may be wider. However, it does not include the corrected Sadowsky func- tional constructed in [33] (although this correction only affects solutions where $|\eta|>1$, so solutions for which $|\eta| \leqslant 1$ everywhere are still spherical). Nor does it include the narrow limit $(w \rightarrow 0)$ of the functional for annular strips derived in [34], nor, seemingly, the functional for narrow residually stressed strips derived in [35]. It would be interesting to find all functionals of the form (2) (or, more generally, functionals with $l=l\left(\kappa, \eta, \kappa^{\prime}, \eta^{\prime}, \ldots\right)$ [28]) with unconstrained spherical solutions, analogous to all functionals with forceless helical solutions having been characterized in [24].

We stress that in this paper we have not considered any constraint on the strip. In particular, the surface of the strip is not required to lie in the surface of the sphere, although solutions, as in Fig. 3(a), that remain close to the equator (i.e., have small geodesic curvature) rotate out of the surface only very little. Strips adhered to a spherical surface (similar to the growing crystals studied in [36]) would obviously have Gaussian curvature $1 / R^{2}$, with $R$ the radius of the sphere. The surface of the strip would then not be developable and therefore not be described by the Sadowsky functional. However, the Sadowsky functional can still be expected to provide a good approximation for the mechanics of a physical ribbon if the stretching energy $U_{s}$ is much smaller than the bending energy $U_{b}$. Now, for an adhered ribbon whose geodesic curvature is much smaller than its normal curvature, we estimate $U_{s} \approx t(w / R)^{4}$ and $U_{b} \approx$ $t^{3} / R^{2}$, where $t$ is the thickness of the ribbon (both energies per unit area). We thus require $w / R \ll t / w$ (in addition to $t / w \ll$ 1 for any ribbon model) and we conclude that the (approximate) validity of the Sadowsky model for such adhered spherical ribbons does not extend to arbitrarily thin ribbons.

\section{ACKNOWLEDGMENT}

We thank an anonymous referee for insightful comments that helped us to improve the paper. 
[1] W. Jiang, J. Chang, J. Jakana, P. Weigele, J. King, and W. Chiu, Structure of epsilon 15 bacteriophage reveals genome organization and DNA packaging/injection apparatus, Nature (London) 439, 612 (2006).

[2] T. Kamasaki, M. Osumi, and I. Mabuchi, Three-dimensional arrangement of F-actin in the contractile ring of fission yeast, J. Cell Biol. 178, 765 (2007).

[3] J. F. Allard and A. D. Rutenberg, Pulling Helices Inside Bacteria: Imperfect Helices and Rings, Phys. Rev. Lett. 102, 158105 (2009).

[4] J. Salje, F. van den Ent, P. de Boer, and J. Loewe, Direct membrane binding by bacterial actin MreB, Mol. Cell 43, 478 (2011).

[5] Q. Yin and X. Shi, Mechanics of rolling of nanoribbon on tube and sphere, Nanoscale 5, 5450 (2013).

[6] J. F. Marko and E. D. Siggia, Stretching DNA, Macromolecules 28, 8759 (1995).

[7] J. F. Marko and E. D. Siggia, Bending and twisting elasticity of DNA, Macromolecules 27, 981 (1994).

[8] J. D. Moroz and P. Nelson, Torsional directed walks, entropic elasticity, and DNA twist stiffness, Proc. Natl. Acad. Sci. 94, 14418 (1997).

[9] L. Giomi and L. Mahadevan, Statistical Mechanics of Developable Ribbons, Phys. Rev. Lett. 104, 238104 (2010).

[10] E. L. Starostin and G. H. M. van der Heijden, Comment on "Statistical Mechanics of Developable Ribbons," Phys. Rev. Lett. 107, 239801 (2011).

[11] E. L. Starostin and G. H. M. van der Heijden, Equilibrium shapes with stress localisation for inextensible elastic Möbius and other strips, J. Elast. 119, 67 (2015).

[12] W. Wunderlich, Über ein abwickelbares Möbiusband, Monatshefte für Mathematik 66, 276 (1962).

[13] M. Sadowsky, Ein elementarer Beweis für die Existenz eines abwickelbaren Möbiusschen Bandes und Zurückführung des geometrischen Problems auf ein Variationsproblem, Sitzungsberichte der Königlich Preußischen Akademie der Wissenschaften zu Berlin 22, 412 (1930).

[14] M. Sadowsky, Theorie der elastisch biegsamen undehnbaren Bänder mit Anwendungen auf das Möbius'sche Band, in A. C. W. Oseen and W. Weibull, Editors, Verhandl. des 3. Intern. Kongr. f. Techn. Mechanik, 1930, Teil II, AB Sveriges Litografiska Tryckerier, 1931, pp. 444-451.

[15] J. Chopin, V. Démery, and B. Davidovitch, Roadmap to the morphological instabilities of a stretched twisted ribbon, J. Elast. 119, 137 (2015).

[16] S. Bartels and P. Hornung, Bending paper and the Möbius strip, J. Elast. 119, 113 (2015).
[17] N. O. Kirby and E. Fried, Gamma-limit of a model for the elastic energy of an inextensible ribbon, J. Elast. 119, 35 (2015).

[18] G. S. Manning, Relaxed elastic line on a curved surface, Quart. Appl. Math. 45, 515 (1987).

[19] J. Guven and P. Vázquez-Montejo, Confinement of semiflexible polymers, Phys. Rev. E 85, 026603 (2012).

[20] A. Huynen, E. Detournay, and V. Denoël, Surface constrained elastic rods with application to the sphere, J. Elast. 123, 203 (2016).

[21] A. J. Spakowitz and Z.-G. Wang, Semiflexible Polymer Confined to a Spherical Surface, Phys. Rev. Lett. 91, 166102 (2003).

[22] J. Langer and D. A. Singer, Lagrangian aspects of the Kirchhoff elastic rod, SIAM Rev. 38, 605 (1996).

[23] L. Mahadevan and J. B. Keller, The shape of a Möbius band, Proc. R. Soc. London A 440, 149 (1993).

[24] M. Barros and A. Ferrández, On the energy density of helical proteins, J. Math. Biol. 69, 1801 (2014).

[25] R. Perline, A class of vortex filament solitons in fluids, plasmas, and superconductors, J. Math. Phys. 51, 103519 (2010).

[26] R. E. Goldstein and D. M. Petrich, Solitons, Euler's Equation, and Vortex Patch Dynamics, Phys. Rev. Lett. 69, 555 (1992).

[27] G. L. Lamb, Solitons on moving space curves, J. Math. Phys. 18, 1654 (1977).

[28] E. L. Starostin and G. H. M. van der Heijden, Force and moment balance equations for geometric variational problems on curves, Phys. Rev. E 79, 066602 (2009).

[29] D. Chubelaschwili and U. Pinkall, Elastic strips, Manuscripta Mathematica 133, 307 (2010).

[30] T. Hangan, Elastic strips and differential geometry, Rend. Sem. Mat. Univ. Pol. Torino 63, 179 (2005).

[31] P. Hornung, Euler-Lagrange equations for variational problems on space curves, Phys. Rev. E 81, 066603 (2010).

[32] Y.-C. Wong, A global formulation of the condition for a curve to lie on a sphere, Monatshefte für Mathematik 67, 363 (1963).

[33] L. Freddi, P. Hornung, M. G. Mora, and R. Paroni, A corrected Sadowsky functional for inextensible elastic ribbons, J. Elast. 123, 125 (2016).

[34] M. A. Dias and B. Audoly, "Wunderlich, meet Kirchhoff": A general and unified description of elastic ribbons and thin rods, J. Elast. 119, 49 (2015).

[35] E. Efrati, Non-Euclidean ribbons: Generalized Sadowsky functional for residually-stressed thin and narrow bodies, J. Elast. 119, 251 (2015).

[36] G. Meng, J. Paulose, D. R. Nelson, and V. N. Manoharan, Elastic instability of a crystal growing on a curved surface, Science 343, 634 (2014). 